\title{
Heuristic solutions to robust variants of the minimum-cost integer flow problem
}

\author{
Marko Špoljarec \\ Privredna banka Zagreb \\ Radnička cesta 44, 10000 Zagreb, Croatia \\ E-mail: marko.spoljarec@pbz.hr \\ Robert Manger \\ University of Zagreb, Faculty of Science, Department of Mathematics \\ Bijenička cesta 30, 10000 Zagreb, Croatia \\ E-mail: manger@math.hr
}

\begin{abstract}
This paper deals with robust optimization applied to network flows. Two robust variants of the minimum-cost integer flow problem are considered. Thereby, uncertainty in problem formulation is limited to arc unit costs and expressed by a finite set of explicitly given scenarios. It is shown that both problem variants are NP-hard. To solve the considered variants, several heuristics based on local search or evolutionary computing are proposed. The heuristics are experimentally evaluated on appropriate problem instances.
\end{abstract}

Keywords: robust optimization, network flow, minimum-cost flow, heuristic, local search, evolutionary computing.

\section{Introduction}

Flows in networks are an important modeling paradigm used in optimization. Models based on flows are applied in different areas, such as transport, logistics, production planning, network design, etc. There are several types of network flow problems, but most of them reduce to the relatively general minimum-cost flow problem.

As it is true for any optimization task, an instance of the minimum-cost flow problem is specified by exact values of parameters within its objective function and its constraints. However, in real-world situations parameter values are often hard to determine since they may depend on unpredictable future circumstances or perhaps cannot be measured accurately. Then we speak about uncertainty in problem formulation. The traditional approach to optimization tends to ignore uncertainty. It means that parameters are very often given approximate, unreliable or ad-hoc values. Unfortunately, such approach can lead to inferior or even infeasible solutions. Therefore, instead of ignoring 
uncertainty, it is much better to admit its existence and find a more appropriate way of dealing with it.

A state-of-the-art approach to deal with the mentioned uncertainty is called robust optimization. According to that approach, a finite or infinite set of scenarios is defined each of them specifies a possible combination of parameter values. Only those solutions are considered that are feasible for all scenarios. The behavior of any solution under any scenario is measured in some way. For each solution, its worst behavior over the whole set of scenarios is recorded. As the optimal solution in the robust sense, the one is chosen whose recorded worst behavior happens to be the best among all solutions. A consequence of using the robust approach is that the initial (let us say) minimization problem transforms into a more complex min-max problem. The robust solution does not need to be really optimal for any scenario, but it is chosen in order to be acceptable even in the most adverse circumstances.

The foundations of robust optimization have been laid out in the seminal works [7, 8, 9, 21]. For our purposes the most important reference is the book [21], which provides a framework for robust discrete (or combinatorial) optimization. More recent surveys of the whole discipline and its general results are available in [1, 2, 10, 19].

The existing research papers on robust discrete optimization are mostly concerned with the shortest path problem. Also well covered is the knapsack or the minimum spanning tree problem. There are not too many works dealing with flows in networks. The available papers on robust flows [3, 4, 8, 11, 12, 22, 23, 24, 26, 27, 28] are hard to compare since they use quite different definitions and concepts. Some of them, e.g. [12, 28], capture uncertainty in parameters by assuming a finite and explicitly given set of scenarios. Other papers, e.g. [3, 4, 8, 22, 23, 24, 26], assume intervals or even more general geometric constructs for parameter values, thus dealing implicitly with an infinite but rather regular set of scenarios. There are also big differences regarding the scope or extent of uncertainty.

It is well known that the conventional (non-robust) flow problems can be solved efficiently by polynomial-time algorithms. On the other hand, many robust variants of flow problems turn out to be NP-hard. Consequently, finding efficient algorithms for robust variants is a challenging and important research topic. At this moment, there are not many algorithms found in literature that can be regarded as efficient and suitable for real-world situations. Authors of the available papers have mainly been concerned with complexity issues or specialized solutions. For instance, the authors of [28] propose an algorithm for solving a robust minimum-cost integer flow problem, which relies on an unspecified sub-algorithm for solving the corresponding (NP-hard) robust shortest path problem. As far as we know, there are no reports in literature on solving robust minimum-cost flow problems by standard heuristics or meta-heuristics.

The aim of this paper is to demonstrate that robust flow problems can be solved with reasonable efficiency even if they are NP-hard. The aim is also to present useful and practically relevant algorithms for solving some types of robust flow problems. In order to fulfill its aims, the paper considers two robust variants of the minimumcost integer flow problem. Both of them are based on an explicitly given finite set of scenarios. Uncertainty expressed through scenarios is limited to arc unit costs. It is shown that both problem variants are NP-hard, which is an indication that they could be solved efficiently only by approximate algorithms. The paper proposes several heuristic solutions based on local search and on evolutionary computing, respectively. 
The proposed heuristics are tested on carefully constructed problem instances, and their performance is measured in terms of accuracy and speed.

Our decision to study heuristics instead of some other types of algorithms is motivated by the fact that real-world problem instances may be fairly large. It is true that smaller instances can be solved exactly by general-purpose optimization software packages. Also, moderately sized tasks can be solved at least approximately by relaxation (ignoring integrality constraints) and rounding. Still, very large instances may easily become intractable for general-purpose methods even in the relaxed form, so that they could be tackled only by heuristics.

Apart from this introduction, the rest of the paper is organized as follows. Section 2 specifies two robust variants of the minimum-cost integer flow problem and shows that both of them are NP-hard. Section 3 describes some basic procedures with flows that will further on be used as building blocks for heuristics. Section 4 presents our heuristics for solving the considered problem variants. Section 5 reports on experimental evaluation of the heuristics. The final Section 6 gives conclusions.

\section{Problem variants and their complexity}

We first describe the conventional (non-robust) variant of the minimum-cost integer flow problem. We use the formulation from [25]. Let $G=(V, A)$ be a network (directed graph), where $V=\left\{v_{1}, v_{2}, \ldots, v_{n}\right\}$ is a set of $n$ elements called vertices and $A \subset V \times V$ is a set of ordered pairs of vertices called arcs. Each arc $\left(v_{i}, v_{j}\right)$ is characterized by its capacity $u_{i j}$ and its unit cost $c_{i j}$. Vertex $v_{1}$ is called the source and vertex $v_{n}$ the sink. We consider feasible flows that transfer a required amount of flow $F$ from the source to the sink. Thereby, a feasible flow is a nonnegative function defined on arcs, which conforms to the flow conservation rule in vertices and to the capacity constraints along arcs. The arc flow value assigned to an arc $\left(v_{i}, v_{j}\right)$ is denoted by $x_{i j}$. The flow cost is obtained by summing the products $c_{i j} x_{i j}$ over all arcs $\left(v_{i}, v_{j}\right)$. The objective is to find a feasible flow with minimal cost.

In this paper we assume that all input data $u_{i j}, c_{i j}, F$ are nonnegative integers. Moreover, we expect that the flow itself should consist of integer arc values $x_{i j}$. Thus we indeed consider the minimum-cost integer flow problem. The restriction to integers makes sense when a discrete phenomenon is modeled, such as transportation of packaged goods, assignment of tasks to agents, etc.

The described minimum-cost integer flow problem can more formally be defined as the following integer linear programming problem [25]:

$$
\begin{aligned}
& \mathrm{MCIF} \quad \ldots \quad z=\sum_{\left(v_{i}, v_{j}\right) \in A} c_{i j} x_{i j} \longrightarrow \min \\
& \sum_{\substack{v_{j} \in V \\
\left(v_{i}, v_{j}\right) \in A}} x_{i j}-\sum_{\substack{v_{j} \in V \\
\left(v_{j}, v_{i}\right) \in A}} x_{j i}=\left\{\begin{array}{l}
F \text { if } v_{i}=v_{1} \\
-F \text { if } v_{i}=v_{n} \\
0 \text { otherwise }
\end{array}, \text { for all } v_{i} \in V\right. \\
& 0 \leq x_{i j} \leq u_{i j} \text {, for all }\left(v_{i}, v_{j}\right) \in A \\
& x_{i j} \text { integer, for all }\left(v_{i}, v_{j}\right) \in A
\end{aligned}
$$


Note that the considered minimum-cost flow problem includes also the well-known minimum-cost maximal flow problem [13] as a special case. Indeed, for a given network we can compute its maximal flow value in advance. Then, in order to solve the minimumcost maximal flow problem, we can solve our problem with $F$ set to the computed maximal flow value. Note also that in some literature, e.g. [5, 18, 20], the minimum-cost flow problem is described in a different way allowing more sources and sinks. Although seemingly more general, such alternative formulation can easily be reduced to ours. Reduction is obtained by introducing an additional source and an additional sink, and by adding arcs with appropriate capacities from the new source to each of the original sources, as well as from each of the original sinks to the new sink.

Now we describe two robust variants of the minimum-cost integer flow problem. According to our adopted approach from [21], uncertainty in input data is captured by a finite set of scenarios $S$. A particular scenario $s \in S$ is expressed through a specific set of arc unit costs $c_{i j}^{s}$. We assume that network structure and arc capacities are the same for all scenarios. Consequently, the set of feasible flows also remains the same, no matter which scenario has been chosen.

The first robust variant is called absolute [21] or min-max [1] robust variant. There, the behavior of a feasible flow under a certain scenario is measured absolutely, i.e. as the actual flow cost. For each feasible flow, its worst behavior (i.e. its maximal flow cost) over all scenarios is recorded. As the robust solution, the flow is chosen whose worst behavior is the best (i.e. minimal) among all feasible flows. More formally, the absolute robust variant is defined as follows:

$$
\begin{aligned}
& \mathrm{RMCIF}-\mathrm{A} \quad \ldots \quad z=\max _{s \in S}\left\{\sum_{\left(v_{i}, v_{j}\right) \in A} c_{i j}^{s} x_{i j}\right\} \longrightarrow \min \\
& \text { subject to: } \\
& \sum_{\substack{v_{j} \in V \\
\left(v_{i}, v_{j}\right) \in A}} x_{i j}-\sum_{\substack{v_{j} \in V \\
\left(v_{j}, v_{i}\right) \in A}} x_{j i}=\left\{\begin{array}{l}
F \text { if } v_{i}=v_{1} \\
-F \text { if } v_{i}=v_{n} \\
0 \text { otherwise }
\end{array}, \text { for all } v_{i} \in V\right. \\
& 0 \leq x_{i j} \leq u_{i j} \text {, for all }\left(v_{i}, v_{j}\right) \in A \\
& x_{i j} \text { integer, for all }\left(v_{i}, v_{j}\right) \in A
\end{aligned}
$$

The second robust variant is called robust deviation [21] or robust min-max regret [1] variant. There, the behavior of a feasible flow under a certain scenario $s$ is measured as deviation of the actual cost from the optimal cost $z^{s}$ for that scenario (computed in advance). Again, the flow is chosen whose worst behavior over the whole set of scenarios is the best possible. Or more formally, the robust deviation variant is defined in the following way:

$$
\operatorname{RMCIF-D} \quad \ldots \quad z=\max _{s \in S}\left\{\sum_{\left(v_{i}, v_{j}\right) \in A} c_{i j}^{s} x_{i j}-z^{s}\right\} \rightarrow \min
$$

subject to: 


$$
\begin{aligned}
& \sum_{\substack{v_{j} \in V \\
\left(v_{i}, v_{j}\right) \in A}} x_{i j}-\sum_{\substack{v_{j} \in V \\
\left(v_{j}, v_{i}\right) \in A}} x_{j i}=\left\{\begin{array}{l}
F \text { if } v_{i}=v_{1} \\
-F \text { if } v_{i}=v_{n} \\
0 \text { otherwise }
\end{array}, \text { for all } v_{i} \in V\right. \\
& 0 \leq x_{i j} \leq u_{i j}, \text { for all }\left(v_{i}, v_{j}\right) \in A \\
& x_{i j} \text { integer, for all }\left(v_{i}, v_{j}\right) \in A
\end{aligned}
$$

On the first sight, RMCIF-A and RMCIF-D seem to be nonlinear since their objective functions involve min-max combinations. However, both problems can easily be transformed into integer linear programming problems. Transformation is done by introducing an additional variable $y$ in a manner shown in [21]. Indeed, here are the corresponding "linearized" versions RMCIF-A' and RMCIF-D', respectively:

$$
\begin{aligned}
& \text { RMCIF-A' } \quad \ldots \quad y \longrightarrow \min \\
& \text { subject to: } \\
& \sum_{\left(v_{i}, v_{j}\right) \in A} c_{i j}^{s} x_{i j} \leq y, \text { for all } s \in S \\
& \sum_{\substack{v_{j} \in V \\
\left(v_{i}, v_{j}\right) \in A}} x_{i j}-\sum_{\substack{v_{j} \in V \\
\left(v_{j}, v_{i}\right) \in A}} x_{j i}=\left\{\begin{array}{l}
F \text { if } v_{i}=v_{1} \\
-F \text { if } v_{i}=v_{n} \\
0 \text { otherwise }
\end{array}, \text { for all } v_{i} \in V\right. \\
& 0 \leq x_{i j} \leq u_{i j} \text {, for all }\left(v_{i}, v_{j}\right) \in A \\
& x_{i j} \text { integer, for all }\left(v_{i}, v_{j}\right) \in A \\
& \mathrm{RMCIF}^{\prime} \mathrm{D}^{\prime} \quad \ldots \quad y \longrightarrow \min \\
& \text { subject to: } \\
& \sum_{\left(v_{i}, v_{j}\right) \in A} c_{i j}^{s} x_{i j} \leq y+z^{s}, \text { for all } s \in S \\
& \sum_{\substack{v_{j} \in V \\
\left(v_{i}, v_{j}\right) \in A}} x_{i j}-\sum_{\substack{v_{j} \in V \\
\left(v_{j}, v_{i}\right) \in A}} x_{j i}=\left\{\begin{array}{l}
F \text { if } v_{i}=v_{1} \\
-F \text { if } v_{i}=v_{n} \\
0 \text { otherwise }
\end{array}, \text { for all } v_{i} \in V\right. \\
& 0 \leq x_{i j} \leq u_{i j} \text {, for all }\left(v_{i}, v_{j}\right) \in A \\
& x_{i j} \text { integer, for all }\left(v_{i}, v_{j}\right) \in A
\end{aligned}
$$

Note that our two robust variants RMCIF-A and RMCIF-D differ only in the way how the robust objective function is defined. In some contexts we will treat both variants together by referring simply to the "robust minimum-cost integer flow problem". We will also use the common acronym RMCIF.

To illustrate the introduced problem variants, we give now a simple example. Let us consider the network shown in Figure 1. All arc capacities are set to 1 . Vertex $v_{1}$ is the source and vertex $v_{14}$ the sink. The desired flow value is $F=2$. Arc unit costs 
are given by two alternative scenarios and shown as arc labels separated by "/". We would like to compute the solution of the conventional variant MCIF for each scenario. Also, we would like to solve the absolute robust variant RMCIF-A, as well as the robust deviation variant RMCIF-D.

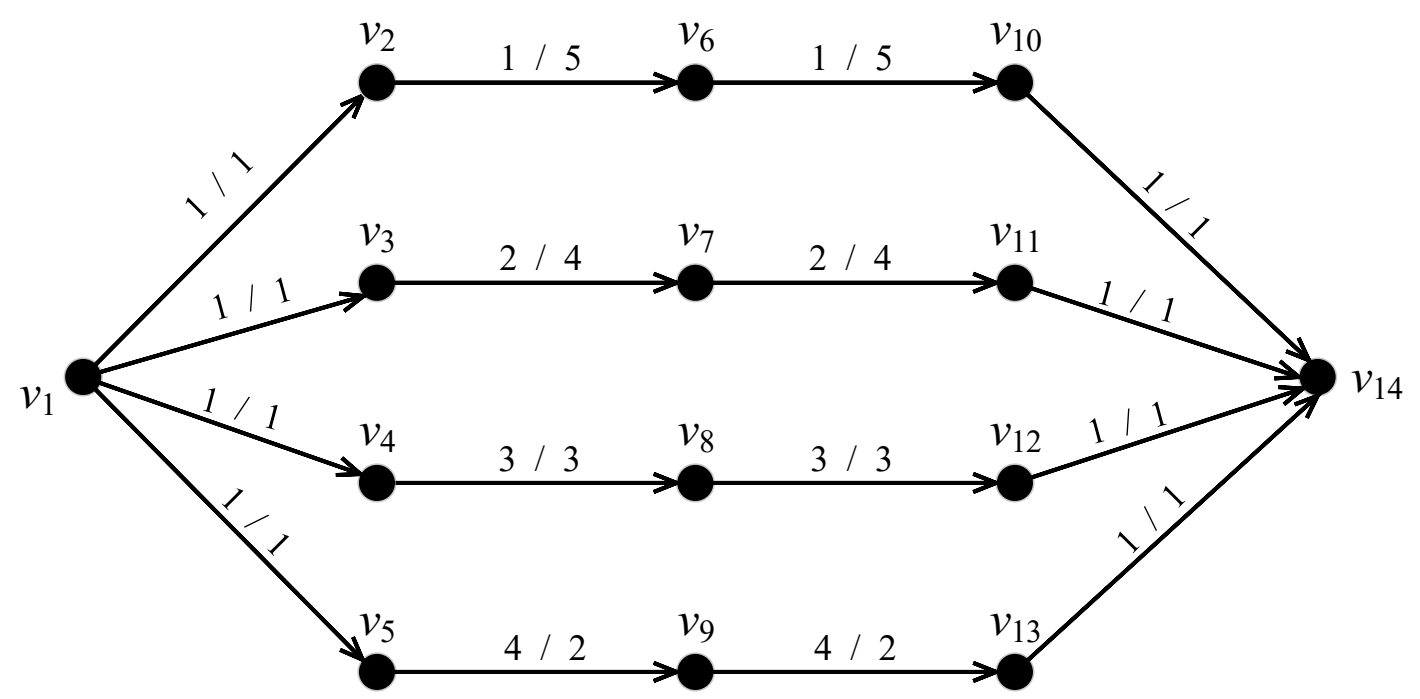

Figure 1: A sample problem instance with two scenarios.

As we can see, our network in Figure 1 consists of four separate paths. Each path connects the source to the sink and has capacity 1 . Thus any feasible flow with value $F=2$ must be a combination of exactly two unit flows, each of them sending one unit of flow through a distinct path.

Let flow 1 be the flow through the uppermost path in Figure 1, flow 2 through the next uppermost path, ..., flow 4 through the lowermost path. By examining all six combinations of two among four flows, we can easily check that the optimal solution under the first scenario is the combination of flow 1 with flow 2 - the respective cost is 10. Similarly, the optimal solution under the second scenario is flow 3 plus flow 4 with the cost 14 . On the other hand, the combination of flow 2 with flow 4 gives the optimal solution in the sense of absolute robustness and its cost is 16. Finally, the optimum in the sense of robust deviation can be achieved either by flow 1 plus flow 4, or by flow 2 plus flow 3 - the optimal deviation of cost is 4 .

The presented example clearly shows that a robust solution can differ from any conventional solution corresponding to a particular scenario. Also, we see that the considered two criteria of robustness can produce different results.

Now we will discuss computational complexity issues [16, 25] regarding different variants of the minimum-cost integer flow problem. It is well-known [18] that the conventional (non-robust) variant MCIF can be solved in polynomial time, even if integral solutions are required. However, by switching to robust versions, the problem becomes much more complex, as stated by the following proposition.

Proposition 1 The problem variants RMCIF-A and RMCIF-D are both NP-hard. This claim is true even if the number of scenarios is limited to 2. 
The proof is obtained by polynomial reduction [16, 25] of suitable shortest path problem variants to our considered flow problem variants. Namely, the standard source-to-sink shortest path problem is equivalent to the minimum-cost integer flow problem posed in the same network, with arc lengths interpreted as unit costs, and with the required flow value $F=1$. Indeed, for $F=1$, due to integrality, each flow must be a unit flow. Any unit flow determines a path from the source to the sink, and vice-versa. Thus the minimum-cost unit flow is equivalent to the shortest path. Moreover, the solution of any robust variant of the minimum-cost unit flow problem is equivalent to the solution of the corresponding robust variant of the shortest path problem. On the other hand, it has been proved in [21] that both robust variants of the shortest path problem are NP-hard, even in the case with only 2 scenarios. Therefore the corresponding network flow variants must also be NP-hard.

In spite of the observed NP-hardness, smaller instances of RMCIF-A or RMCIF-D can be solved exactly by general-purpose integer programming software packages. Thereby, "linearized" formulations RMCIF-A' and RMCIF-D', respectively, should be used. Still, larger problem instances can easily become intractable. The only practical way of dealing with such larger instances is switching to approximate solutions.

\section{Basic procedures with flows}

In this section we present some basic computational procedures dealing with flows. Most of them are relatively well known since they are incorporated within standard networking algorithms. However, our intention is to use them in a novel way as building blocks for heuristics. The procedures are listed and specified in Table 1. It is assumed that all involved flows operate on the same network.

In the remaining part of this section we briefly describe how the procedures from Table 1 can be implemented. In many cases, implementation is based on finding a path in a network. Depending on a particular procedure, here follow the details.

- Summation of flows is implemented in a straightforward way. The same is true for flow centering.

- To implement flow decomposition, we consider the network consisting of arcs that are used by $f$, i.e. arcs whose arc flow values according to $f$ are $>0$. Within that network we find a path from the source to the sink, e.g. with Moore's BFS algorithm [18]. The found path determines the first unit flow $\phi_{1}$. We subtract $\phi_{1}$ from $f$, thus obtaining a simpler integer flow $f_{1}$ with value $F-1$. We repeat the same routine on $f_{1}$ in order to obtain the second unit flow $\phi_{2}, \ldots$, etc., until all $F$ unit flows are found.

- Flow augmentation is achieved in the following way. We construct the so-called displacement or residual network associated with $f$ [13, 18, 20]. Such network consists of forward arcs (showing where $f$ can be increased) and of backward arcs (indicating where $f$ can be decreased). We try to find an arbitrary path in the displacement network from the source to the sink. For this purpose we use e.g. Moore's BFS or Tarjan's DFS algorithm [18]. If the sought path does not exist, then $F$ cannot be increased since it is already maximal. Otherwise, in order to 


\begin{tabular}{|c|c|c|}
\hline $\begin{array}{l}\text { Procedure } \\
\text { name }\end{array}$ & Input & Output \\
\hline $\begin{array}{l}\text { Summation } \\
\text { of flows }\end{array}$ & $\begin{array}{l}\text { A list of flows } \\
f_{1}, f_{2}, \ldots, f_{r}\end{array}$ & $\begin{array}{l}\text { The pseudo-flow } g \text { equal } \\
\text { to the sum of } f_{1}, f_{2}, \ldots, f_{r}\end{array}$ \\
\hline $\begin{array}{l}\text { Flow } \\
\text { decomposition }\end{array}$ & $\begin{array}{l}\text { An integer flow } f \\
\text { with value } F\end{array}$ & $\begin{array}{l}\text { A list } \phi_{1}, \phi_{2}, \ldots, \phi_{F} \text { of } F \text { unit } \\
\text { flows whose sum is equal to } f\end{array}$ \\
\hline $\begin{array}{l}\text { Flow } \\
\text { centering }\end{array}$ & $\begin{array}{l}\text { A list of flows } \\
f_{1}, f_{2}, \ldots, f_{r}\end{array}$ & $\begin{array}{l}\text { The (possibly non-integer) flow } \\
g \text { equal to the arithmetic mean } \\
\text { of } f_{1}, f_{2}, \ldots, f_{r}\end{array}$ \\
\hline $\begin{array}{l}\text { Flow } \\
\text { augmentation }\end{array}$ & $\begin{array}{l}\text { An integer flow } f \\
\text { with value } F\end{array}$ & $\begin{array}{l}\text { An integer flow } g \text { with value } \\
>F, \text { which can be considered } \\
\text { as an augmented version of } f\end{array}$ \\
\hline $\begin{array}{l}\text { Flow } \\
\text { rounding }\end{array}$ & $\begin{array}{l}\text { A non-integer flow } \\
f \text { with value } F\end{array}$ & $\begin{array}{l}\text { An integer flow } g, \text { which can } \\
\text { be regarded as an integral } \\
\text { approximation of } f \text {, and } \\
\text { whose value is } F \text { rounded } \\
\text { to the nearest integer }\end{array}$ \\
\hline $\begin{array}{l}\text { Flow } \\
\text { composition }\end{array}$ & $\begin{array}{l}\text { Two lists of unit } \\
\text { flows, } \phi_{1}, \phi_{2}, \ldots, \phi_{F} \\
\text { and } \psi_{1}, \psi_{2}, \ldots, \psi_{F}\end{array}$ & $\begin{array}{l}\text { An integer flow } h \text {, which is a } \\
\text { sum of roughly equal number } \\
\text { of unit flows from both input } \\
\text { lists, and whose value is } F\end{array}$ \\
\hline $\begin{array}{l}\text { Flow cost } \\
\text { reduction }\end{array}$ & $\begin{array}{l}\text { A scenario } s, \text { an } \\
\text { integer flow } f \text { with } \\
\text { value } F \text { whose cost } \\
\text { under scenario } s \\
\text { is } C\end{array}$ & $\begin{array}{l}\text { An integer flow } g \text {, which can be } \\
\text { considered as a cost-reduced } \\
\text { version of } f \text {, having the same } \\
\text { value } F \text { but cost (under } \\
\text { scenario } s \text { ) smaller than } C\end{array}$ \\
\hline $\begin{array}{l}\text { Flow } \\
\text { perturbation }\end{array}$ & $\begin{array}{l}\text { An integer flow } f \\
\text { with value } F\end{array}$ & $\begin{array}{l}\text { An integer flow } g \text { with the same } \\
\text { value } F \text {, which can be regarded } \\
\text { as a slightly perturbed version } \\
\text { of } f\end{array}$ \\
\hline $\begin{array}{l}\text { Flow } \\
\text { harmonization }\end{array}$ & $\begin{array}{l}\text { Two integer flows } \\
f \text { and } g \text {, both } \\
\text { with value } F\end{array}$ & $\begin{array}{l}\text { An integer flow } h \text { with the same } \\
\text { value } F \text {, which can be regarded } \\
\text { as an adjusted version of } f \\
\text { being more "similar" to } g \\
\text { than the original version }\end{array}$ \\
\hline $\begin{array}{l}\text { Finding a } \\
\text { flow with a } \\
\text { given value }\end{array}$ & An integer $F$ & $\begin{array}{l}\text { An arbitrary integer flow } f \\
\text { with value } F\end{array}$ \\
\hline $\begin{array}{l}\text { Finding a } \\
\text { minimum-cost } \\
\text { flow }\end{array}$ & $\begin{array}{l}\text { An integer } F \text {, } \\
\text { a scenario } s\end{array}$ & $\begin{array}{l}\text { An integer flow } f \text { with value } F \text {, } \\
\text { whose cost (under scenario } s \text { ) } \\
\text { is minimal }\end{array}$ \\
\hline
\end{tabular}

Table 1: Specification of our basic procedures with flows. 
obtain $g$, we modify $f$ according to the found path, so that the same integral amount of flow is added to each forward arc and subtracted from the inverse of each backward arc. The used amount of flow is chosen so that it does not exceed the limit imposed by arc capacities along the path.

- To implement flow rounding, we consider the pseudo-flow $\bar{f}$ obtained from $f$ by rounding all its arc values $x_{i j}$ to $\left\lfloor x_{i j}+0.5\right\rfloor$ (i.e. to the nearest integer). Note that $\bar{f}$ may violate the conservation rule in vertices, but it still must obey the capacity constraints along arcs due to the fact that all arc capacities are integral. We construct the network consisting of arcs whose arc flow values according to $\bar{f}$ are $>0$. In that network we try to find a path from the source to the sink, by employing e.g. Moore's BFS or Tarjan's DFS algorithm [18. The found path determines the first unit flow $\phi_{1}$. We subtract $\phi_{1}$ from $\bar{f}$, thus obtaining a simpler pseudo-flow $\bar{f}_{1}$ with smaller value. We repeat the same routine on $\bar{f}_{1}$ in order to obtain the second unit flow $\phi_{2}, \ldots$, etc. Iteration stops after $\lfloor F+0.5\rfloor$ steps, or earlier if the remaining network becomes disconnected (i.e. if there is no path from the source to the sink). Then we sum up the collected unit flows $\phi_{1}, \phi_{2}, \ldots$ in order to obtain the first version of $g$. It is obvious that the obtained $g$ must be a feasible integer flow, namely it satisfies the flow conservation rule (since it is a sum of unit flows) and it also obeys the capacity constraints (since its arc values cannot exceed the corresponding values in $\bar{f}$ ). Finally, if the value of $g$ is less than $\lfloor F+0.5\rfloor$, we repeatedly modify $g$ by using the previously described flow augmentation procedure until the desired flow value is reached.

- Flow composition can be done in the following way. A combined list of $\leq F$ unit flows is formed by selecting elements from the first and from the second input list in alternation (a randomly chosen element from the first list, then a randomly chosen element from the second list, then again from the first list, ..., etc.). During the whole construction process, the already selected unit flows must be compatible in the sense that their sum is a feasible flow. If in some step compatibility is violated, then that step may be repeated by randomly choosing some other element from the same input list. However, even with such repeated trials, it can still happen that the combined list cannot be completed to length $F$ because any remaining element from the appropriate input list would lead to incompatibility. In that case, construction stops with the combined list containing $<F$ unit flows. Anyway, the elements from the combined list are summed up in order to produce the first version of the output flow $h$. If the obtained $h$ has the value $<F$, it is repeatedly modified by the flow augmentation procedure until the desired value $F$ is reached.

- To achieve flow cost reduction, we try to find a negative-length cycle in the corresponding displacement network (considering unit costs as arc lengths, assuming that costs of forward arcs have positive signs and costs of backward arcs negative signs). To find such cycle we can use e.g. the Floyd-Warshall algorithm [13, 18, 20]. The remaining details regarding how the found cycle is used to transform $f$ into $g$ are similar as for flow augmentation.

- To realize flow perturbation, we try to find an arbitrary cycle in the corresponding 
displacement network - it can be done e.g. by the backtracking DFS algorithm [18]. The remaining steps needed to obtain $g$ from $f$ are analogous as for flow cost reduction.

- Flow harmonization can be implemented as flow perturbation applied to $f$. However, a customized displacement network associated with $f$ is applied. Namely, only those forward arcs are taken into account that are used by $g$. Also, only those backward arcs are considered that correspond to arcs not used by $g$.

- To find a flow with a given value, we could start from the null flow $f$ and transform it into a flow with value $F$ by repeated application of the flow augmentation procedure. There exist also more compact and more efficient implementations, such as the Edmonds-Karp, Dinic or Malholtra-Kumar-Maheshwari algorithm [18, 20].

- In order to find a minimum-cost flow, we could start from an arbitrary flow $f$ with value $F$, e.g. the one obtained by the previously described procedure. Then we could transform $f$ into a minimum-cost flow by iterating the flow cost reduction procedure. Again, there exist more compact and more efficient implementations. Some of them are based on finding shortest paths in networks (considering arc unit costs as their lengths). Finding shortest paths can be implemented by Dijkstra's algorithm (if arc lengths are nonnegative) or by the Bellman-Ford algorithm (if arc lengths can be negative but there is still no cycle with negative length) [13, 18].

\section{Heuristic solutions}

In this section we first describe our local search algorithm for solving the RMCIF problem. Its outline is specified by the pseudo-code shown in Figure 2. Our pseudo-code follows the well-known overall strategy described e.g. in [25, 29]. Thus for a given problem instance, the algorithm starts by finding a feasible flow that will serve as the initial solution, i.e. the first version of the current solution. Then the current solution is iteratively improved. In each iteration, the so-called neighborhood of the current solution is generated. The neighborhood consists of feasible flows obtained by modifying the current flow in certain ways. All flows in the neighborhood are evaluated according to the chosen robust criterion of optimality (objective function). The best-evaluated member of the neighborhood is identified. If the best-evaluated member is better then the current solution, it becomes the new current solution and the algorithm proceeds with another iteration. Otherwise the algorithm stops and proclaims the last current solution to be nearly optimal (in the robust sense) for the considered problem instance.

As indicated by Figure 2, our local-search algorithm relies on two predefined parameters: the first of them determines the neighborhood size, and the second is used within a stopping condition. We see that the whole search can in fact terminate in two ways: (normally) when the current flow cannot be improved any more, or (exceptionally) when a predefined limit for total number iterations is reached.

According to the outline shown in Figure 2, there are many possible variants of local search. First of all, the variants can differ in the chosen robust criterion of optimality. Indeed, we can choose absolute robustness according to the problem specification 


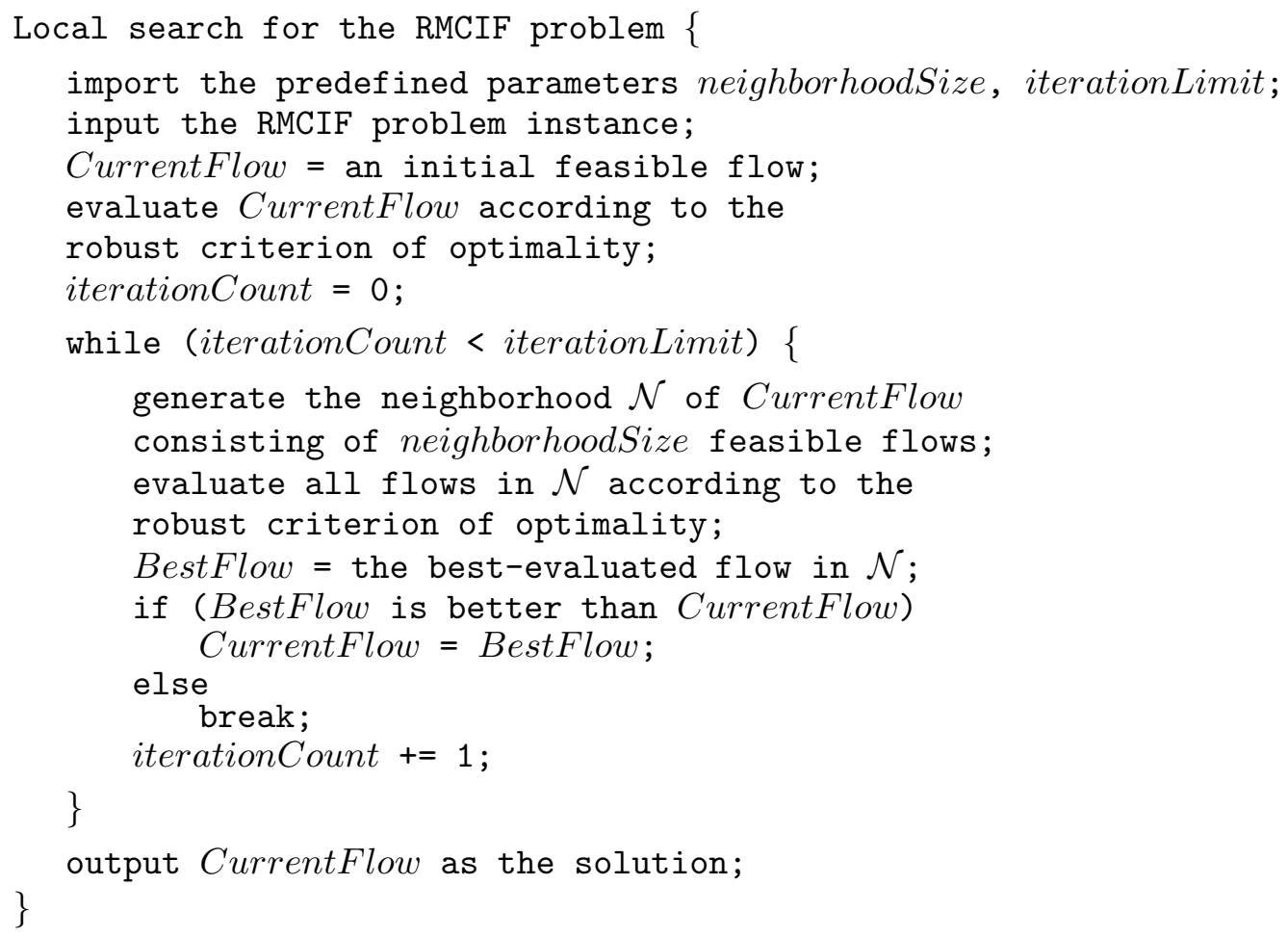

Figure 2: Generic pseudo-code of local search.

RMCIF-A, or robust deviation captured by RMCIF-D. Note that in the case of robust deviation the cost of the minimum-cost flow for each particular scenario is needed within the objective function. Those costs should be computed in advance by using the appropriate procedure from the previous section.

After the robust criterion has been chosen, there is still a lot of possibilities how to construct the initial feasible flow or generate the neighborhood of the current flow. In this paper we restrict to four local-search variants for each robustness criterion. The variants (i.e. heuristics) are called LS-1, LS-2, LS-3 and LS-4, respectively. Their properties are summarized in Table 2 .

As it can be seen from Table 2, our four heuristics based on local search start from different initial flows. However, the neighborhood of a current flow is always generated in the same manner. One of the heuristics involves repeated execution of the pseudo-code from Figure 2. All initial flows and neighborhoods are produced by using appropriate basic procedures from the previous section. Here are some more details.

- In LS-1 the initial flow is constructed in a rather rudimentary way. The idea is to choose an arbitrary feasible flow whose design is not influenced by the given scenarios. It can be obtained by finding a flow with a given value.

- In LS-2 the initial flow is chosen by a kind of greedy approach. First, the minimumcost flow for each scenario is found. Then, the obtained minimum-cost flows are evaluated according to the chosen robust criterion of optimality. Finally, the best-evaluated flow is selected. 


\begin{tabular}{|l||l|l|l|}
\hline & $\begin{array}{l}\text { Initial } \\
\text { flow }\end{array}$ & $\begin{array}{l}\text { Neighborhood } \\
\text { generation }\end{array}$ & $\begin{array}{l}\text { Repeated } \\
\text { execution? }\end{array}$ \\
\hline \hline LS-1 & $\begin{array}{l}\text { arbitrary } \\
\text { feasible flow }\end{array}$ & $\begin{array}{l}\text { iterated flow } \\
\text { cost reduction }\end{array}$ & no \\
\hline LS-2 & $\begin{array}{l}\text { best-evaluated } \\
\text { minimum-cost flow }\end{array}$ & $\begin{array}{l}\text { iterated flow } \\
\text { cost reduction }\end{array}$ & no \\
\hline LS-3 & $\begin{array}{l}\text { rounded centered } \\
\text { minimum-cost flow }\end{array}$ & $\begin{array}{l}\text { iterated flow } \\
\text { cost reduction }\end{array}$ & no \\
\hline LS-4 & $\begin{array}{l}\text { minimum-cost flow for } \\
\text { a particular scenario }\end{array}$ & $\begin{array}{l}\text { iterated flow } \\
\text { cost reduction }\end{array}$ & $\begin{array}{l}\text { yes - once for } \\
\text { each scenario }\end{array}$ \\
\hline
\end{tabular}

Table 2: Variants of local search.

- In LS-3 the initial flow is constructed by using a balanced approach. Again, the minimum-cost flow for each scenario is found. Next, the centered flow based on all those minimum-cost flows is computed. Finally, flow rounding is applied to the centered flow in order to make it integral.

- In LS-4 initialization is subject to multiple trials. The initial flow is again chosen as the minimum-cost flow for a particular scenario. However, the whole pseudocode from Figure 2 is executed separately for each scenario. Finally, among all solutions obtained in this manner the best one (according to the robust criterion) is chosen as the final solution.

- In all considered variants of local search, neighborhood generation is based on flow cost reduction according to various scenarios. Indeed, the current flow is costreduced separately according to each scenario. Thus the neighborhood initially consists of as many flows as there are scenarios. In order to obtain a larger neighborhood, cost reduction for a particular scenario can be iterated several times and all intermediate flows can be recorded.

In the remaining part of this section we describe our evolutionary algorithm for solving the RMCIF problem. Its outline is given by the pseudo-code in Figure 3. Our pseudo-code follows the well-known overall strategy described e.g. in [15, 29]. Indeed, it is a randomized computing process which maintains a population of feasible solutions (flows). The population is iteratively changed, thus producing a series of population versions called generations. All flows in a generation are evaluated according to the chosen robust criterion of optimality (objective function). We expect that the bestevaluated flow in the last generation should represent a nearly optimal solution (in the robust sense) for the considered problem instance.

The most important elements of the algorithm from Figure 3 are the evolutionary operators that produce new flows from old ones, thus changing the current population. There is a unary operator, called mutation, which makes a small change of a single flow. There is also a binary operator, called crossover, which creates a new flow (child) by combining parts of two existing flows (parents). Also an important element of the algorithm is the initialization procedure, which generates the initial population of flows. 


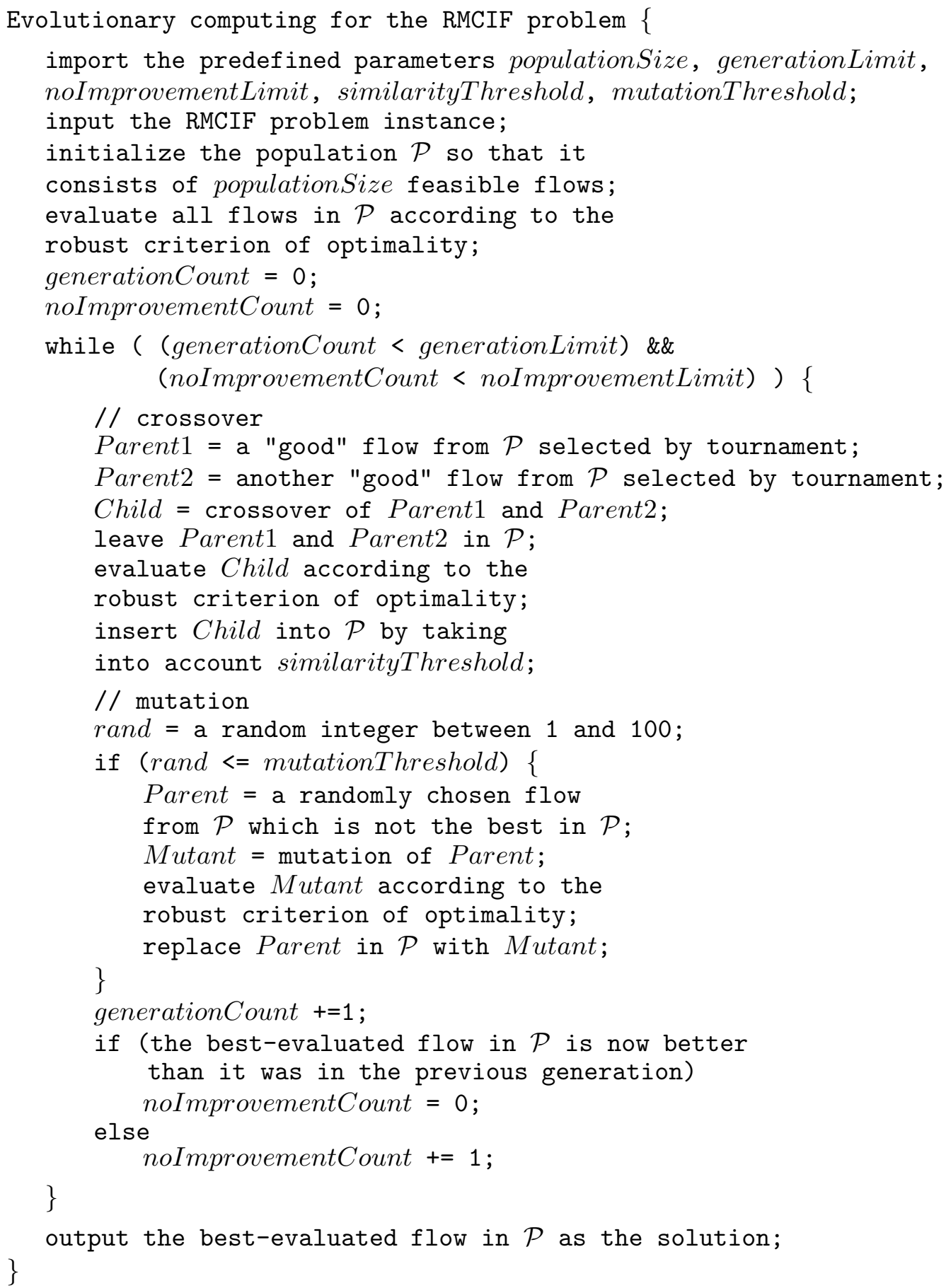

Figure 3: Generic pseudo-code of evolutionary computing. 
Note that evolutionary computing described by Figure 3 relies on five predefined parameters. The first of them determines the population size, and the next two are used within stopping conditions. We see that the whole computation can stop in two ways: either when the total number of generations reaches a predefined limit, or when the best solution does not improve during a predefined number of consecutive generations. The last but one parameter is used within the procedure for inserting children into the population, as it will be explained later. The last parameter controls intensity of using mutation.

An additional element of our evolutionary algorithm is selection of a "good" (or "bad") flow from the population. It is accomplished by so-called tournament selection [15, 29]. Indeed, several flows are picked up randomly, and then the best-evaluated (or the worst-evaluated) among them is selected.

Yet another part of our evolutionary computing is the insertion procedure used to insert newly produced solutions (children) into the current population, while keeping population size constant. As it is suggested by the pseudo-code in Figure 3, our insertion procedure relies on the concept of similarity and uses the predefined parameter called similarityThreshold. We say that two flows are similar if the difference of their robust costs (robust objective function values), expressed as a percentage of the best robust cost within the population, is not greater than similarityThreshold. Insertion "by taking into account similarityThreshold" means the following. If there exists another flow in the population that is similar in the above sense to the new one, then the better of those two "twins" is retained and the other one discarded. If there is no similar flow, then the new one is retained, and some other "bad" flow from the population is selected by tournament and discarded.

The outline shown in Figure 3 allows many possible variants, thus producing slightly different evolutionary heuristics. First of all, the variants can differ in the chosen robust criterion of optimality - it can be absolute robustness according to RMCIF-A or robust deviation specified by RMCIF-D. Again, in the case of robust deviation the minimal flow costs for particular scenarios have to be computed in advance.

After the robust criterion has been chosen, there are still different options regarding how the population is initialized or how the evolutionary operators are implemented. In this paper we study nine evolution variants for each robustness criterion. The variants (heuristics) are called EC-1, EC-2, .., EC-9, respectively. Their properties are presented in Table 3.

As shown in Table 3, our nine heuristics based on evolutionary computing use essentially the same method to initialize the population. However, they employ three different crossover operators and three mutation operators. All initializations, crossovers and mutations are realized by combining basic procedures from the previous section. Here are some details.

- In EC-1, EC-2 and EC-3 the crossover of two flows $f$ and $g$ is obtained in a relatively straightforward way. First, the centered version of $f$ and $g$ is computed. Then, flow rounding of the centered flow is performed in order to achieve integrality. The rounded centered flow is output as the child.

- In EC-4, EC-5 and EC-6 the crossover of two flows $f$ and $g$ is obtained in a more subtle way. Namely, the child is produced by flow harmonization applied to $f$ and $g$. 


\begin{tabular}{|l||l|l|l|}
\hline & $\begin{array}{l}\text { Initial } \\
\text { population }\end{array}$ & $\begin{array}{l}\text { Crossover } \\
\text { operator }\end{array}$ & $\begin{array}{l}\text { Mutation } \\
\text { operator }\end{array}$ \\
\hline \hline EC-1 & $\begin{array}{l}\text { mutated } \\
\text { minimum-cost flows }\end{array}$ & $\begin{array}{l}\text { flow centering, } \\
\text { flow rounding }\end{array}$ & $\begin{array}{l}\text { flow } \\
\text { perturbation }\end{array}$ \\
\hline EC-2 & $\begin{array}{l}\text { mutated } \\
\text { minimum-cost flows }\end{array}$ & $\begin{array}{l}\text { flow centering, } \\
\text { flow rounding }\end{array}$ & $\begin{array}{l}\text { flow cost } \\
\text { reduction }\end{array}$ \\
\hline EC-3 & $\begin{array}{l}\text { mutated } \\
\text { minimum-cost flows }\end{array}$ & $\begin{array}{l}\text { flow centering, } \\
\text { flow rounding }\end{array}$ & $\begin{array}{l}\text { local } \\
\text { search }\end{array}$ \\
\hline EC-4 & $\begin{array}{l}\text { mutated } \\
\text { minimum-cost flows }\end{array}$ & $\begin{array}{l}\text { flow } \\
\text { harmonization }\end{array}$ & $\begin{array}{l}\text { flow } \\
\text { perturbation }\end{array}$ \\
\hline EC-5 & $\begin{array}{l}\text { mutated } \\
\text { minimum-cost flows }\end{array}$ & $\begin{array}{l}\text { flow } \\
\text { harmonization }\end{array}$ & $\begin{array}{l}\text { flow cost } \\
\text { reduction }\end{array}$ \\
\hline EC-6 & $\begin{array}{l}\text { mutated } \\
\text { minimum-cost flows }\end{array}$ & $\begin{array}{l}\text { flow } \\
\text { harmonization }\end{array}$ & $\begin{array}{l}\text { local } \\
\text { search }\end{array}$ \\
\hline EC-7 & $\begin{array}{l}\text { mutated } \\
\text { minimum-cost flows }\end{array}$ & $\begin{array}{l}\text { flow decomposition, } \\
\text { flow composition }\end{array}$ & $\begin{array}{l}\text { flow } \\
\text { perturbation }\end{array}$ \\
\hline EC-8 & $\begin{array}{l}\text { mutated } \\
\text { minimum-cost flows }\end{array}$ & $\begin{array}{l}\text { flow decomposition, } \\
\text { flow composition }\end{array}$ & $\begin{array}{l}\text { flow cost } \\
\text { reduction }\end{array}$ \\
\hline EC-9 & $\begin{array}{l}\text { mutated } \\
\text { minimum-cost flows }\end{array}$ & $\begin{array}{l}\text { flow decomposition, } \\
\text { flow composition }\end{array}$ & $\begin{array}{l}\text { local } \\
\text { search }\end{array}$ \\
\hline
\end{tabular}

Table 3: Variants of evolutionary computing.

- In EC-7, EC-8 and EC-9 the crossover of two flows $f$ and $g$ is constructed in a rather complex way. The construction starts with flow decomposition, which breaks both $f$ and $g$ into lists of unit flows. Those two lists are then recombined through flow composition in order to produce the child flow.

- In EC-1, EC-4 and EC-7 the mutant of a flow $f$ is obtained by applying flow perturbation to $f$.

- In EC-2, EC-5 and EC-8 the mutant of a flow $f$ is obtained by applying flow cost reduction to $f$ according to a randomly chosen scenario $s$.

- In EC-3, EC-6 and EC-9 mutation is implemented as a full-scale local search. Thus the mutant of a flow $f$ is produced by running the whole local search algorithm from Figure 2 with $f$ taken as the initial solution. Consequently, EC-3, EC-6 and EC-9 can be regarded as hybrids of evolution and local search.

- In all evolution variants, the initial population is generated in the following way. First, the minimum-cost flow for each scenario is constructed. All obtained flows, being optimal for particular scenarios, are inserted into the population. If such population happens to be too small, additional members are produced from the original ones by applying mutation. Thereby the same mutation operator is used as in the main part of the algorithm. Even more population members can be produced by successive mutation, or by collecting intermediate solutions if mutation is implemented by local search. 


\section{$5 \quad$ Experimental results}

In order to perform experiments, we have implemented our four variants of local search and nine variants of evolutionary computing (altogether thirteen heuristics) as a single C\# program [30]. Each particular heuristic has been assembled from basic procedures according to Table 2 or 3 from Section 4. The basic procedures themselves have been implemented as suggested in Section 3. Thereby the following standard networking algorithms [18, 20] have been employed:

- the Malholtra-Kumar-Maheshvari algorithm for finding flows with maximal or given values,

- Moore's BFS algorithm for finding paths with minimum number of arcs,

- Dijskstra's and the Bellman-Ford algorithm for finding shortest paths,

- the Floyd-Warshall algorithm for finding negative-length cycles,

- the backtracking DFS algorithm for finding arbitrary cycles.

The implemented high-level algorithms for local search and evolutionary computing follow the generic pseudo-codes from Figures 2 and 3, respectively. All parameters from the pseudo-codes are supported. The program is always configured with certain fixed values of those parameters, but at any time it can easily be reconfigured with some other values.

The configured program takes as input the specification of a concrete RMCIF problem instance, i.e.

- the set of vertices $V$ and the set of $\operatorname{arcs} A$ with arc capacities $u_{i j}$,

- the desired flow value $F$,

- the set of scenarios $S$ with $\operatorname{arc}$ unit $\operatorname{costs} c_{i j}^{s}$ for each scenario,

- the minimal flow costs $z^{s}$ for particular scenarios computed in advance.

The given RMCIF problem instance is solved repeatedly:

- according to both problem variants, RMCIF-A and RMCIF-D, respectively,

- with all thirteen heuristics, i.e. LS-1, LS-2, .., LS-4, EC-1, EC-2, .., EC-9.

For each combination of problem variant and heuristic the program produces the following output:

- full specification of the solution, i.e. the list of arc flow values $x_{i j}$ and the value of the robust objective function,

- time in seconds taken by the program to produce the solution. 
Our program has been installed on a standard notebook computer with a $2.60 \mathrm{GHz}$ Intel Core i5-6440HQ processor and 4 GBytes of memory. After some preliminary testing, the program parameters have been set in the following way: neighborhoodSize $=30$, iterationLimit $=\infty$, populationSize $=30$, generationLimit $=\infty$, noImprovementLimit $=300$, similarityThreshold $=5$, mutation Threshold $=1$.

In order to measure accuracy of approximate solutions obtained by heuristics, we have also implemented an exact solver for the RMCIF problem. It is based on the wellknown general-purpose optimization package IBM ILOG CPLEX [17]. Thanks to that additional software, it was possible to obtain exact solutions (i.e. solutions that are truly optimal in the robust sense) for smaller problem instances. Thereby the "linearized" problem versions RMCIF-A' and respectively RMCIF-D' were solved. The exact solver takes similar input data as our heuristics, produces as output the exact robust solution costs for both robustness criteria, and measures its own computing time during each computation. It has been installed on the same hardware as the $\mathrm{C} \#$ program that runs heuristics.

In our experiments, we have used a set of 30 carefully constructed RMCIF problem instances, whose identifiers are I-01, I-02, . ., I-30, respectively. They have been chosen as large enough to be nontrivial, but still small enough to be solvable by the exact solver. It means that slightly larger instances (more vertices, arcs or scenarios) cannot anymore be solved to optimality, at least not with our hardware and software. Namely, on such larger instances the exact solver fails due to memory overflow.

All our problem instances involve layered networks similar to those occurring in standard applications of network flows. The idea is illustrated by Figure 4 . Thus all vertices except the source and the sink are distributed among layers. Arcs can connect only vertices between adjacent layers. Layer width (number of vertices within a layer) can be fixed (the same for each layer) or varying.
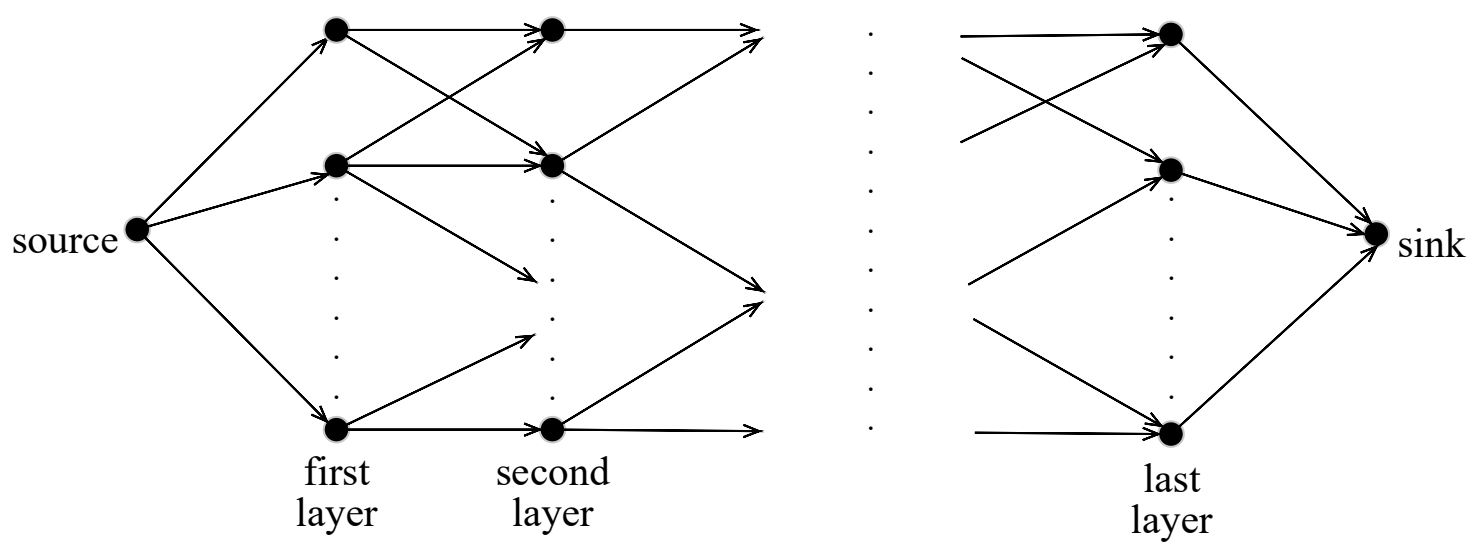

Figure 4: Structure of a layered network.

Important properties of our problem instances are summarized in Table 4 . The values shown in the table have been chosen by hand, and the remaining details not visible from the table (such as actual configuration of arcs or actual values of $u_{i j}$ or $c_{i j}^{s}$ ) have been generated randomly. The complete specification of each problem instance can be obtained upon a request from the first author by e-mail. 
Regarding our choice of problem instances, one may argue that it would be much better if we used some standard benchmark data instead of our own synthetic data. This is certainly true. However, the obstacle is that, to the best of our knowledge, a suitable benchmark collection does not exist. There are some well-known repositories of minimum-cost flow problem instances available on Internet, e.g. [6, 14], but they deal only with the conventional (non-robust) problem variant. Expanding a conventional benchmark instance with more scenarios would not make sense since it would produce a completely new instance that cannot be regarded as a benchmark any more.

In our experiments we have run our $\mathrm{C} \#$ program on all problem instances from Table 4. Consequently, each problem instance has been solved according to each of the two robust optimality criteria by each of the thirteen heuristics. The obtained solutions have been stored in a data file, which is too voluminous to be reproduced here but is again available from the first author by e-mail. Still, some excerpts from that file are visible in Table 5. Indeed, for each problem instance and each robustness criterion the table presents a selected approximate solution. It is the one with the smallest robust cost among those whose computing time is at most half of the time needed by the exact solver. To allow comparison, the table also contains the corresponding exact solutions. Any solution, either exact or approximate, is described by its robust cost (a whole number) and its computing time in seconds (a decimal number). In case of an approximate solution, the involved heuristic is also identified.

The raw measurements from the described experiments could be processed in many different ways. In the remaining part of this section we present a simple analysis based on the whole collection of values from our data file. Within our analysis we have computed the relative error of each approximate solution, as the difference between the approximate robust cost and the corresponding exact cost divided by the exact cost. Also, we have computed the speedup of each approximate computation as the quotient of the corresponding "exact" computing time vs. the "approximate" computing time. The obtained relative errors for a given robust problem variant and a given heuristic have been averaged over the whole set of problem instances. The same kind of averaging has also been done with the speedups. All averages obtained in this way are presented in Table 6 . The table reveals "typical" behavior of each heuristic applied to each problem variant.

Now follows a discussion about the obtained results. The first thing we can immediately observe is a big difference between the RMCIF-A and the RMCIF-D problem variant. Although the formulations of both variants look similar, they behave quite differently. From Table 5 it is visible that "exact" computing times for RMCIF-D are usually much larger than for RMCIF-A, which means that RMCIF-D is harder to solve exactly. From Table 6 it is visible that relative errors for RMCIF-D are about 4 times larger than for RMCIF-A, which means that RMCIF-D is also harder to approximate. Larger relative errors can partially be explained by the following fact: the objective function in RMCIF-D measures deviations from conventional costs, not actual costs, and takes therefore smaller values than the objective function in RMCIF-A. Consequently, a certain difference from the exact solution within RMCIF-D will produce a larger relative error than it would produce within RMCIF-A. 


\begin{tabular}{|c|c|c|c|c|c|c|c|}
\hline $\begin{array}{c}\text { Instance } \\
\text { identifier }\end{array}$ & $\begin{array}{c}\text { Number of } \\
\text { vertices } \\
|V|\end{array}$ & $\begin{array}{c}\text { Number } \\
\text { of arcs } \\
|A|\end{array}$ & $\begin{array}{c}\text { Number of } \\
\text { scenarios } \\
|S|\end{array}$ & $\begin{array}{c}\text { Flow } \\
\text { value } \\
F\end{array}$ & $\begin{array}{c}\text { Number } \\
\text { of } \\
\text { layers }\end{array}$ & $\begin{array}{c}\text { Layer } \\
\text { width }\end{array}$ & $\begin{array}{c}\text { Range } \\
\text { for } \\
u_{i j}, c_{i j}^{s}\end{array}$ \\
\hline \hline I-01 & 18 & 80 & 30 & 243 & 2 & 8 (fixed) & $0-99$ \\
\hline I-02 & 18 & 56 & 30 & 177 & 4 & 4 (fixed) & $0-99$ \\
\hline I-03 & 18 & 32 & 30 & 93 & 8 & 2 (fixed) & $0-99$ \\
\hline I-04 & 18 & 34 & 30 & 20 & 7 & $3,2,2,2,2,2,3$ & $0-99$ \\
\hline I-05 & 17 & 60 & 30 & 177 & 3 & 5 (fixed) & $0-99$ \\
\hline I-06 & 17 & 42 & 30 & 134 & 5 & 3 (fixed) & $0-99$ \\
\hline I-07 & 17 & 59 & 30 & 198 & 2 & 11,4 & $0-99$ \\
\hline I-08 & 24 & 143 & 15 & 456 & 2 & 11 (fixed) & $0-99$ \\
\hline I-09 & 24 & 44 & 15 & 48 & 11 & 2 (fixed) & $0-99$ \\
\hline I-10 & 24 & 130 & 15 & 104 & 3 & $10,9,3$ & $0-99$ \\
\hline I-11 & 23 & 112 & 15 & 270 & 3 & 7 (fixed) & $0-99$ \\
\hline I-12 & 23 & 60 & 15 & 52 & 7 & 3 (fixed) & $0-99$ \\
\hline I-13 & 23 & 72 & 15 & 34 & 3 & $7,3,11$ & $0-99$ \\
\hline I-14 & 27 & 110 & 10 & 216 & 5 & 5 (fixed) & $0-99$ \\
\hline I-15 & 27 & 153 & 10 & 78 & 3 & $3,8,14$ & $0-99$ \\
\hline I-16 & 26 & 168 & 10 & 506 & 2 & 12 (fixed) & $0-99$ \\
\hline I-17 & 26 & 144 & 10 & 270 & 3 & 8 (fixed) & $0-99$ \\
\hline I-18 & 26 & 120 & 10 & 228 & 4 & 6 (fixed) & $0-99$ \\
\hline I-19 & 26 & 88 & 10 & 229 & 6 & 4 (fixed) & $0-99$ \\
\hline I-20 & 26 & 69 & 10 & 112 & 8 & 3 (fixed) & $0-99$ \\
\hline I-21 & 26 & 48 & 10 & 108 & 12 & 2 (fixed) & $0-99$ \\
\hline I-22 & 26 & 118 & 10 & 16 & 5 & $2,4,6,8,4$ & $0-99$ \\
\hline I-23 & 30 & 224 & 5 & 628 & 2 & 14 (fixed) & $0-99$ \\
\hline I-24 & 30 & 161 & 5 & 247 & 4 & 7 (fixed) & $0-99$ \\
\hline I-25 & 30 & 104 & 5 & 218 & 7 & 4 (fixed) & $0-99$ \\
\hline I-26 & 30 & 56 & 5 & 63 & 14 & 2 (fixed) & $0-99$ \\
\hline I-27 & 30 & 198 & 5 & 97 & 3 & $7,10,11$ & $0-99$ \\
\hline I-28 & 29 & 180 & 5 & 356 & 3 & 9 (fixed) & $0-99$ \\
\hline I-29 & 29 & 78 & 5 & 97 & 9 & 3 (fixed) & $0-99$ \\
\hline I-30 & 29 & 90 & 5 & 30 & 7 & $2,5,3,4,6,2,5$ & $0-99$ \\
\hline
\end{tabular}

Table 4: Properties of the chosen RMCIF problem instances. 


\begin{tabular}{|c|c|c|c|c|}
\hline $\begin{array}{l}\text { Instance } \\
\text { identifier }\end{array}$ & $\begin{array}{l}\text { RMCIF-A } \\
\text { exact } \\
\text { solution }\end{array}$ & $\begin{array}{l}\text { RMCIF-A } \\
\text { approximate } \\
\text { solution }\end{array}$ & $\begin{array}{l}\text { RMCIF-D } \\
\text { exact } \\
\text { solution }\end{array}$ & $\begin{array}{l}\text { RMCIF-D } \\
\text { approximate } \\
\text { solution }\end{array}$ \\
\hline$\overline{\mathrm{I}-01}$ & \begin{tabular}{|ll}
39700 & $5.86 \mathrm{~s}$
\end{tabular} & \begin{tabular}{lll|} 
EC-9 & 40510 & $2.79 \mathrm{~s}$ \\
\end{tabular} & 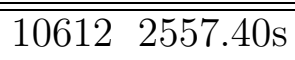 & $\begin{array}{lll}\text { EC-7 } & 11115 & 3.37 \mathrm{~s}\end{array}$ \\
\hline$\overline{\mathrm{I}-02}$ & \begin{tabular}{|ll}
47676 & $29.23 \mathrm{~s}$ \\
\end{tabular} & $\begin{array}{|lll|}\text { EC-8 } & 48228 & 1.25 \mathrm{~s}\end{array}$ & $12746404.24 \mathrm{~s}$ & $\begin{array}{lll}\text { EC-9 } & 13507 & 1.99 \mathrm{~s}\end{array}$ \\
\hline $\mathrm{I}-03$ & $52501 \quad 0.20 \mathrm{~s}$ & $\begin{array}{lll}\text { EC-6 } & 52642 & 0.06 \mathrm{~s} \\
\end{array}$ & $0.29 \mathrm{~s}$ & \begin{tabular}{|ll} 
EC-2 & 5291 \\
\end{tabular} \\
\hline $\mathrm{I}-04$ & $0.25 \mathrm{~s}$ & \begin{tabular}{lll|} 
EC-8 & 9487 & $0.11 \mathrm{~s}$ \\
\end{tabular} & $0.45 \mathrm{~s}$ & \begin{tabular}{|ll} 
EC-3 & 835 \\
\end{tabular} \\
\hline $\mathrm{I}-05$ & $\begin{array}{|ll|}37864 & 62.78 \mathrm{~s} \\
\end{array}$ & $\begin{array}{|lll|}\text { EC-9 } & 38420 & 1.79 \mathrm{~s}\end{array}$ & $103081545.33 \mathrm{~s}$ & $\begin{array}{lll}\text { EC-7 } & 10771 & 1.29 \mathrm{~s}\end{array}$ \\
\hline $\mathrm{I}-06$ & \begin{tabular}{|ll}
43074 & $0.56 \mathrm{~s}$
\end{tabular} & $\begin{array}{|lll|}\text { EC-6 } & 44823 & 0.16 \mathrm{~s} \\
\end{array}$ & $26.88 \mathrm{~s}$ & EC-8 $10580 \quad 1.86 \mathrm{~s}$ \\
\hline $\mathrm{I}-07$ & 32636 & $\begin{array}{|lll|}\text { EC-9 } & 32938 & 2.25 \mathrm{~s} \\
\end{array}$ & $48.87 \mathrm{~s}$ & $\begin{array}{lll}\text { EC-9 } & 11185 \quad 2.40 \mathrm{~s}\end{array}$ \\
\hline $\mathrm{I}-08$ & 68343 & $\begin{array}{lll}\text { EC-4 } & 70835 & 0.15 \mathrm{~s} \\
\end{array}$ & $16302 \quad 320.35 \mathrm{~s}$ & \begin{tabular}{|lll} 
EC-7 & 17340 & $18.97 \mathrm{~s}$ \\
\end{tabular} \\
\hline I-09 & 30351 & $\begin{array}{lll}\text { EC-1 } & 31223 & 0.05 \mathrm{~s} \\
\end{array}$ & $0.39 \mathrm{~s}$ & \begin{tabular}{|ll} 
EC-8 & 6121 \\
\end{tabular} \\
\hline $\mathrm{I}-10$ & 18790 & \begin{tabular}{lll|} 
EC-8 & 19750 & $1.77 \mathrm{~s}$ \\
\end{tabular} & $217.35 \mathrm{~s}$ & \begin{tabular}{|ll} 
EC-7 & 9226
\end{tabular} \\
\hline $\mathrm{I}-11$ & $49736 \quad 101.12 \mathrm{~s}$ & $\begin{array}{lll}\text { EC-9 } & 52057 & 9.45 \mathrm{~s} \\
\end{array}$ & $14987 \quad 485.17 \mathrm{~s}$ & $\begin{array}{|lll|}\text { EC-9 } & 16155 & 8.32 \mathrm{~s} \\
\end{array}$ \\
\hline $\mathrm{I}-12$ & 19576 & \begin{tabular}{lll|} 
EC-8 & 20327 & $0.47 \mathrm{~s}$ \\
\end{tabular} & $1.15 \mathrm{~s}$ & \begin{tabular}{|ll} 
EC-7 & 9761 \\
\end{tabular} \\
\hline $\mathrm{I}-13$ & $0.25 \mathrm{~s}$ & $\begin{array}{|lll|}\text { EC-1 } & 8160 & 0.07 \mathrm{~s} \\
\end{array}$ & $0.67 \mathrm{~s}$ & \begin{tabular}{|ll} 
EC-8 & 974 \\
\end{tabular} \\
\hline $\mathrm{I}-14$ & $59609 \quad 22.40 \mathrm{~s}$ & \begin{tabular}{lll|} 
EC-8 & 61452 & $6.31 \mathrm{~s}$ \\
\end{tabular} & $68.27 \mathrm{~s}$ & $\begin{array}{|lll|}\text { EC-8 } & 19671 & 6.77 \mathrm{~s} \\
\end{array}$ \\
\hline $\mathrm{I}-15$ & 15388 & $\begin{array}{lll}\text { LS-2 } & 16199 & 0.04 \mathrm{~s} \\
\end{array}$ & $9.97 \mathrm{~s}$ & \begin{tabular}{|ll} 
EC-9 & 6317 \\
\end{tabular} \\
\hline $\mathrm{I}-16$ & 81323 & \begin{tabular}{lll|} 
EC-4 & 85928 & $0.07 \mathrm{~s}$ \\
\end{tabular} & $77.70 \mathrm{~s}$ & $\begin{array}{|lll|}\text { EC-8 } & 16328 & 29.02 s \\
\end{array}$ \\
\hline $\mathrm{I}-17$ & $\begin{array}{|ll|}49328 & 61.03 \mathrm{~s} \\
\end{array}$ & $\begin{array}{|lll|}\text { EC-8 } & 52194 & 8.95 \mathrm{~s} \\
\end{array}$ & $16579800.00 \mathrm{~s}$ & \begin{tabular}{|lll} 
EC-9 & 18626 & $10.37 s$
\end{tabular} \\
\hline $\mathrm{I}-18$ & $\begin{array}{|ll|}53075 & 10.06 \mathrm{~s} \\
\end{array}$ & \begin{tabular}{lll|} 
EC-7 & 54322 & $4.19 \mathrm{~s}$ \\
\end{tabular} & \begin{tabular}{|ll}
16128 & $729.75 \mathrm{~s}$ \\
\end{tabular} & $\begin{array}{|lll|}\text { EC-9 } & 17166 & 9.17 \mathrm{~s} \\
\end{array}$ \\
\hline $\mathrm{I}-19$ & 69116 & $\begin{array}{lll}\text { EC-8 } & 72031 & 2.82 \mathrm{~s} \\
\end{array}$ & $18.12 \mathrm{~s}$ & \begin{tabular}{|lll} 
EC-7 & 22301 & $6.95 \mathrm{~s}$
\end{tabular} \\
\hline $\mathrm{I}-20$ & 48256 & \begin{tabular}{lll|} 
EC-4 & 52440 & $0.03 \mathrm{~s}$ \\
\end{tabular} & 12369 & $\begin{array}{|lll|}\text { EC-7 } & 13765 & 1.29 \mathrm{~s} \\
\end{array}$ \\
\hline $\mathrm{I}-21$ & 69159 & $\begin{array}{lll}\text { EC-5 } & 70111 & 0.04 \mathrm{~s} \\
\end{array}$ & $0.32 \mathrm{~s}$ & \begin{tabular}{|lll} 
EC-2 & 10499 & $0.08 s$
\end{tabular} \\
\hline $\mathrm{I}-22$ & $1.91 \mathrm{~s}$ & \begin{tabular}{lll|} 
EC-7 & 4347 & $0.16 \mathrm{~s}$ \\
\end{tabular} & $1.07 \mathrm{~s}$ & \begin{tabular}{|ll} 
EC-9 & 2124 \\
\end{tabular} \\
\hline $\mathrm{I}-23$ & \begin{tabular}{|l|l|}
75982 \\
\end{tabular} & $\begin{array}{|lll|}\text { EC-5 } & 80466 & 0.16 \mathrm{~s} \\
\end{array}$ & 12821 & \begin{tabular}{|lll} 
EC-6 & 17979 & $0.75 \mathrm{~s}$
\end{tabular} \\
\hline $\mathrm{I}-24$ & 49509 & $\begin{array}{lll}\text { EC-4 } & 54611 & 0.12 s \\
\end{array}$ & 16227 & $\begin{array}{|lll|}\text { EC-5 } & 22624 & 0.09 \mathrm{~s} \\
\end{array}$ \\
\hline $\mathrm{I}-25$ & 76696 & \begin{tabular}{lll|} 
EC-5 & 85025 & $0.07 \mathrm{~s}$ \\
\end{tabular} & 15115 & \begin{tabular}{|lll} 
EC-5 & 20752 & $0.16 \mathrm{~s}$
\end{tabular} \\
\hline $\mathrm{I}-26$ & 46485 & $\begin{array}{lll}\text { EC-4 } & 47452 & 0.02 \mathrm{~s} \\
\end{array}$ & $0.29 \mathrm{~s}$ & \begin{tabular}{|ll}
$\mathrm{LS}-2$ & 7211 \\
\end{tabular} \\
\hline $\mathrm{I}-27$ & 17451 & $\begin{array}{lll}\text { EC-5 } & 18668 & 0.08 \mathrm{~s} \\
\end{array}$ & $1.34 \mathrm{~s}$ & \begin{tabular}{|ll} 
EC-5 & 5216 \\
\end{tabular} \\
\hline $\mathrm{I}-28$ & 66375 & \begin{tabular}{lll|} 
LS-3 & 72481 & $0.03 \mathrm{~s}$ \\
\end{tabular} & 13037 & $\begin{array}{|lll|}\text { EC-5 } & 17504 & 0.11 \mathrm{~s} \\
\end{array}$ \\
\hline $\mathrm{I}-29$ & 44382 & $\begin{array}{lll}\text { EC-4 } & 46395 & 0.04 s \\
\end{array}$ & $0.42 \mathrm{~s}$ & $\begin{array}{|lll|}\text { EC-5 } & 11250 & 0.06 \mathrm{~s} \\
\end{array}$ \\
\hline $\mathrm{I}-30$ & 11269 & $\begin{array}{lll}\text { EC-5 } & 11737 & 0.04 \mathrm{~s} \\
\end{array}$ & $0.43 \mathrm{~s}$ & $\begin{array}{|lll|}\text { EC-7 } & 3302 & 0.20 \mathrm{~s} \\
\end{array}$ \\
\hline
\end{tabular}

Table 5: Solutions for the used RMCIF problem instances. 


\begin{tabular}{|c||cc|cc|}
\hline \multicolumn{1}{|c||}{} & \multicolumn{2}{c|}{ RMCIF-A } & \multicolumn{2}{c|}{ RMCIF-D } \\
\hline \hline LS-1 & $14.40 \%$ & $362.02 \times$ & $56.89 \%$ & $25025.52 \times$ \\
\hline LS-2 & $9.47 \%$ & $500.43 \times$ & $41.04 \%$ & $13789.07 \times$ \\
\hline LS-3 & $10.80 \%$ & $232.98 \times$ & $34.14 \%$ & $10122.76 \times$ \\
\hline LS-4 & $8.01 \%$ & $26.27 \times$ & $35.62 \%$ & $489.01 \times$ \\
\hline EC-1 & $8.98 \%$ & $52.94 \times$ & $34.48 \%$ & $1389.52 \times$ \\
\hline EC-2 & $8.89 \%$ & $43.89 \times$ & $32.83 \%$ & $975.39 \times$ \\
\hline EC-3 & $7.49 \%$ & $17.54 \times$ & $29.44 \%$ & $584.88 \times$ \\
\hline EC-4 & $7.13 \%$ & $169.01 \times$ & $35.86 \%$ & $4901.75 \times$ \\
\hline EC-5 & $7.31 \%$ & $151.64 \times$ & $28.55 \%$ & $5013.30 \times$ \\
\hline EC-6 & $6.35 \%$ & $40.90 \times$ & $29.26 \%$ & $1409.51 \times$ \\
\hline EC-7 & $3.85 \%$ & $4.81 \times$ & $15.17 \%$ & $93.53 \times$ \\
\hline EC-8 & $3.64 \%$ & $4.60 \times$ & $14.49 \%$ & $107.70 \times$ \\
\hline EC-9 & $3.77 \%$ & $3.14 \times$ & $13.18 \%$ & $59.02 \times$ \\
\hline
\end{tabular}

Table 6: Average errors (\%) and average speedups $(\times)$ obtained for different combinations of heuristics vs. problem variants.

The next important thing we can observe from our results is that local search is in general faster but less accurate than evolutionary computing. This is just what anybody would expect. However, there are some fine differences in behavior of both types of heuristics regarding the two problem variants.

Let us restrict for a moment to the part of Table 6 dealing with RMCIF-A. Then we can see that local search applied to that problem variant can obtain moderate precision (errors about 8\%), while evolutionary computing can reach higher precision (errors about 4\%). Among different variants of local search, the most accurate is LS-4. This is not a surprise since LS-4 is the only one with repeated execution, i.e. it repeats the whole searching process several times with different initial flows. On the other hand, LS-4 is for the same reason the slowest among local-search variants, although it still achieves a significant speedup (about $26 \times$ ) compared to exact solving. Among different variants of evolutionary computing, the most accurate are EC-7, EC-8 and EC-9. This is also not a surprise since EC-7, EC-8 and EC-9 are again much more elaborate compared to the remaining evolutionary variants. Namely, their crossover operator is based on full decomposition of given flows into unit flows and composition of mutually compatible unit flows into a new flow, which is time-consuming. Moreover, the mutation operator in EC-9 is a full-scale local search, which is also relatively timeconsuming. As a consequence, EC-7, EC-8 and EC-9 turn out to be rather slow, i.e. they are in average only 3 to 5 times faster than the exact solver. If we are looking for a tradeoff among accuracy and speed, we should use a similar evolutionary variant EC-6, whose accuracy is not much lower (errors about 6\%), but whose speedup is higher (over $40 \times)$.

Let us finally restrict to the part of Table 6 dealing with RMCIF-D. Here, the relative errors are worse than for RMCIF-A, but the speedups are better. Relative ranking of particular heuristics remains roughly the same as for RMCIF-A. A small difference in ranking of local-search variants is that LS-3 now seems to be slightly more accurate than LS-4. Also, among the tree previously established most accurate evolutionary variants, 
EC-9 now appears to be slightly better than the other two. Precision of local search must be regarded as unsatisfactory (errors greater than 34\%). Precision of the best evolutionary variant EC-9 is better, but not spectacular (errors about 13\%). A good news is that EC-9, although being quite slow, now achieves a good speedup (about $60 \times$ ) with respect to the exact solver.

\section{Conclusions}

In this paper we have considered two robust variants of the minimum-cost integer flow problem, i.e the absolute robust (min-max) and the robust deviation (min-max regret) variant, respectively. Uncertainty in problem formulation has been restricted to arc unit costs, and expressed by explicitly given scenarios. Both problem variants turn out to be NP-hard, which justifies their approximate solving.

As approximate solutions to the considered problem variants, thirteen heuristics have been proposed, four of them based on local search, and nine on evolutionary computing. All heuristics have experimentally been evaluated on a set of problem instances big enough to be nontrivial but still small enough to be solved exactly.

According to the obtained experimental results, there is a significant difference among the two considered problem variants. Although both of them look similar on the first sight, the second one is much harder to solve exactly, and even harder to approximate. Therefore the results obtained for the absolute robust variant are more satisfactory than those obtained for the robust deviation variant.

From the obtained experimental results we can also see that the heuristics based on local search are fast but not very accurate. Luckily enough, better precision is assured by evolutionary computing. Among nine evolutionary heuristics, the best results are achieved by the one whose crossover operator is based on flow decomposition/composition and whose mutation operator is in fact improvement by local search. Such heuristic can as well be regarded as a hybrid of evolutionary computing and local search.

A drawback of our most-precise heuristic is its slowness. Indeed, for smaller absolute robust problem instances it is not much faster than an exact algorithm. Still, its speedup improves and becomes satisfactory for larger instances. Consequently, we believe that our most-accurate heuristic will show its full potential on very large instances, i.e. in situations where exact algorithms (or even their relaxed counterparts) fail.

In our future research, we plan to evaluate our heuristics on much larger problem instances. Also, our plan is to extend our solutions to more general minimum-cost integer flow problem variants, which would allow uncertainty in arc capacities as well as in arc unit costs. A third direction of further research would be to consider solving the same problems with other meta-heuristics, such as simulated annealing or particle swarm optimization.

\section{Acknowledgement}

This work has been fully supported by Croatian Science Foundation under the project IP-2018-01-5591. 


\section{References}

[1] Aissi H., Bazgan C., Vanderpooten D., "Min-max and min-max regret versions of combinatorial optimization problems: A survey", European Journal of Operational Research, Vol 197 (2009), 427-438.

[2] Aissi H., Bazgan C., Vanderpooten D., "General approximation schemes for minmax (regret) versions of some (pseudo-)polynomial problems", Discrete Optimizaton, Vol 7 (2010), 136-148.

[3] Aissi H., Vanderpooten D., "Robust capacity expansion of a network under demand uncertainty: a bi-objective approach", Networks, Vol 68 (2016), 185-199.

[4] Atamtürk A., Zhang M., "Two-stage robust network flow and design under demand uncertainty". Operations Research, Vol 55 (2007), 662-673.

[5] Bazaraa M.S., Jarvis J.J., Sherali H.D., Linear Programming and Network Flows, Fourth Edition. Wiley, Hoboken NJ, 2010.

[6] Beasley J.E., OR-Library, Brunel University London, 2018. http://people.brunel.ac.uk/ mastjjb/jeb/info.html. Access 17 Dec 2018.

[7] Ben-Tal A., El Ghaoui L., Nemirovski A., Robust Optimization, Princeton University Press, Princeton NJ, 2009.

[8] Bertsimas, D., Sim M., "Robust discrete optimization and network flows", Mathematical Programming, Vol 98 (2003), 49-71.

[9] Bertsimas D., Sim M., "The price of robustness", Operations Research, Vol 52 (2004), 35-53.

[10] Bertsimas D., Brown D.B., Caramanis C., "Theory and applications of robust optimization", SIAM Review, Vol 53 (2011), 464-501.

[11] Bertsimas D., Nasrabadi E., Stiller S., "Robust and adaptive network flows". Operations Research, Vol 61 (2013), 1218-1242.

[12] Boginski V.L., Commander C.W., Turko T., "Polynomial-time identification of robust network flows under uncertain arc failures", Optimization Letters, Vol 3 (2009), 461-473.

[13] Carre B., Graphs and Networks, Oxford University Press, Oxford UK, 1979.

[14] DIMACS - Center for Discrete Mathematics and Theoretical Computer Science, DIMACS Implementation Challenges, Rutgers University, Piscataway NJ, 2017. http://archive.dimacs.rutgers.edu/Challenges/. Access 17 Dec 2018.

[15] Eiben A.E., Smith J.E., Introduction to Evolutionary Computing, Second edition, Natural Computing Series, Springer, Berlin, 2015.

[16] Garey M.R., Johnson D.S, Computers and Intractability: A Guide to the Theory of NP-Completness, W.H. Freeman, San Francisco CA, 1979. 
[17] IBM Corporation, IBM ILOG CPLEX Optimization Studio, CPLEX User's Manual, Version 12, Release 8, IBM Knowledge Center, 2016.

https://www.ibm.com/support/knowledgecenter/SSSA5P_12.8.0.

Accessed 17 December 2018.

[18] Jungnickel D., Graphs, Networks and Algorithms, Fourth Edition, Springer, Berlin, 2013.

[19] Kasperski A., Zielinski P, "Robust discrete optimization under discrete and interval uncertainty: A survey". In: Doumpos M., Zopounidis C., Grigoroudis E. (editors), Robustness Analysis in Decision Aiding, Optimization, and Analytics, Springer, Cham CH, 2016, pp 113-143.

[20] Korte B., Vygen J., Combinatorial Optimization - Theory and Algorithms, Fifth Edition, Springer, Berlin, 2012.

[21] Kouvelis P., Yu G., Robust Discrete Optimization and its Applications, Springer, Berlin, 1997.

[22] Minoux M., "On robust maximum flow with polyhedral uncertainty sets", Optimization Letters, Vol 3 (2009), 367-376.

[23] Minoux M., "Robust network optimization under polyhedral demand uncertainty is NP-hard", Discrete Applied Mathematics, Vol 158 (2010), 597-603.

[24] Ordonez F., Zhao J., "Robust capacity expansion of network flows". Networks, Vol 50 (2007), 136-145.

[25] Papadimitrou C.H., Steiglitz K., Combinatorial Optimization - Algorithms and Complexity, Dover Publications, Mineola NY, 1998.

[26] Poss M., "A comparison of routing sets for robust network design", Optimization Letters, Vol 8 (2014), 1619-1635.

[27] Righetto G.M., Morabito R., Alem D., "A robust optimization approach for cash flow management in stationery companies", Computers and Industrial Engineering, Vol 99 (2016), 137-152.

[28] Rui M., Jinfu Z., "Robust discrete optimization for the minimum cost flow problem". In: Wang C., Ye Z. (editors), Proceedings of the International Workshop on Intelligent Systems and Applications - ISA 2009, Wuhan, China, 23-24 May 2009. IEEE, Piscataway NJ, 2009.

[29] Talbi E-G., Metaheuristics - From Design to Implementation, Wiley, Hoboken NJ, 2009.

[30] Troelsen A., Japikse P., C\# 6.0 and the .NET 4.6 Framework, Seventh Edition, Apress, New York NY, 2016. 